\title{
Separation of Poly(styrene-block-t-butyl methacrylate) Copolymers by Various Liquid Chromatography Techniques
}

\author{
Tina Šmigovec Ljubič, ${ }^{1}$ David Pahovnik, ${ }^{1}$ Majda Žigon, ${ }^{1,2,3}$ and Ema Žagar ${ }^{1,4}$ \\ ${ }^{1}$ Laboratory for Polymer Chemistry and Technology, National Institute of Chemistry, Hajdrihova 19, \\ 1000 Ljubljana, Slovenia \\ ${ }^{2}$ Center of Excellence for Polymer Materials and Technologies, Tehnološki Park 24, 1000 Ljubljana, Slovenia \\ ${ }^{3}$ University College for Polymer Technology, Glavni trg 1, 2380 Slovenj Gradec, Slovenia \\ ${ }^{4}$ EN-FIST Centre of Excellence, Dunajska 156, 1000, Ljubljana, Slovenia \\ Correspondence should be addressed to Ema Žagar, ema.zagar@ki.si
}

Received 25 October 2011; Accepted 24 November 2011

Academic Editors: D. M. Milojkovic-Opsenica and M. Sajewicz

Copyright () 2012 Tina Šmigovec Ljubič et al. This is an open access article distributed under the Creative Commons Attribution License, which permits unrestricted use, distribution, and reproduction in any medium, provided the original work is properly cited.

\begin{abstract}
The separation of a mixture of three poly(styrene-block-t-butyl methacrylate) copolymers (PS- $b$-P $t$ BMA), consisting of polystyrene (PS) blocks of similar length and $t$-butyl methacrylate (PtBMA) blocks of different lengths, was performed using various chromatographic techniques, that is, a gradient liquid chromatography on reversed-phase (C18 and C8) and normalphase columns, a liquid chromatography under critical conditions for polystyrene as well as a fully automated two-dimensional liquid chromatography that separates block copolymers by chemical composition in the first dimension and by molar mass in the second dimension. The results show that a partial separation of the mixture of PS- $b$-P $t$ BMA copolymers can be achieved only by gradient liquid chromatography on reversed-phase columns. The coelution of the two block copolymers is ascribed to a much shorter PtBMA block length, compared to the PS block, as well as a small difference in the length of the PtBMA block in two of these copolymers, which was confirmed by SEC-MALS and NMR spectroscopy.
\end{abstract}

\section{Introduction}

Over the past few decades block copolymers have attracted significant scientific and economic interest since they are able to self-assemble into long-range-ordered structures in bulk, while in selective solvents they form micellar aggregates with a core-shell structure in which the insoluble block forms the core and the soluble block forms the surrounding corona. Stability and an ability to vary the core-shell dimensions make block copolymers widely applicable in various fields, for example, for colloidal stabilization, the compatibilization of polymer blends, controlled drug delivery, water purification, gene therapy, phase-transfer catalysis, viscosity, and surface modifications [1]. Block copolymers can show a distribution in more than one property, that is, chemical composition, functionality, architecture, and molar mass, and as such they are considered as complex polymers. By using conventional methods of polymer characterization, such as
IR or NMR, only the average structural features of copolymers without their distributions can be determined [2]. The chemical composition distribution (CCD) and functionality distribution of copolymers can be determined by liquid chromatography (LC) based on different mechanisms of separation, for example, liquid-adsorption chromatography (LAC), which is a gradient method and liquid chromatography under critical conditions (LCCC), which is an isocratic method [3]. LAC separation is directed by enthalpic interactions, while in LCCC the enthalpic interactions are compensated by entropy losses of the solute [4].

The molar-mass averages and distributions of polymers are usually determined using size-exclusion chromatography (SEC), which is governed by entropic effects and results in the elution of macromolecules according to their hydrodynamic volume. SEC in combination with different detectors (e.g., UV/VIS, IR) also gives information about the chemical composition as a function of the molar mass. However, only 
the average composition at a certain molecular size can be determined. For high-throughput screening and analysis, short and wide-bore columns were developed, making a high-speed separation possible [5].

By combining different LC techniques (LAC or LCCC with SEC) into a two-dimensional chromatographic system, it is possible to correlate a certain composition (or functionality) with the molar mass $[6,7]$. The optimal analysis time in two-dimensional liquid chromatography (2D-LC) is achieved if the second dimension is as short as possible, while giving sufficient resolution, which is fulfilled by wide-bore high-speed SEC columns. The complete transfer of fractions between both dimensions is possible using a software-controlled automatic valve equipped with two sample loops. Automatization of the 2D-LC system has resulted in numerous applications of 2D separations [8-11].

Living polymerization techniques (e.g., anionic polymerization) allow the synthesis of well-defined polymers. For the synthesis of functional (co)polymers, protected monomers with masked functional groups have been employed. For example, amphiphilic poly(styrene-block-methacrylic acid) copolymers (PS- $b$-PMAA) have been prepared by the deprotection of the protected carboxylic groups of poly(styreneblock- $t$-butyl methacrylate) copolymers (PS- $b$-P $t$ BMA). The PS- $b$-PMAA can self-assemble into polymeric micelles and/or vesicles with nanometre-sized dimensions while being dissolved in a selective solvent for one of the blockstypically water. These copolymers have been employed as materials in semiconductors, nanoparticles, micelleencapsulated carbon nanotubes, and so forth. However, since all application fields require a well-defined structure and molar mass of the PS- $b$-PMAA copolymers various 2DLC techniques have been developed for their simultaneous determination [12].

The diblock PS- $b$-PtBMA copolymers were widely studied by Glöckner et al. [13, 14] using gradient liquid chromatography on different stationary phases, for example, bare silica gel, phenyl, and octadecyl columns. On the silica gel column with the isooctane/THF mobile phase the separation of PS- $b$-P $t$ BMA copolymers was not efficient, while it was possible to separate admixed PS from the copolymer. In contrast, the separation of PS- $b$-PtBMA copolymers was successful on phenyl or octadecyl stationary phases with methanol $(\mathrm{MeOH}) / \mathrm{THF}$ gradients. The retention volume decreased with increasing PtBMA content in the block copolymer. This was in contrast to the elution of PS- $b$ PMMA in the gradient elution on a silica stationary phase where the retention was increased by increasing the content of the PMMA in the copolymer, since this block is more polar than the PtBMA block. The inversed elution order was explained by the bulky $t$-butyl group, which shields the polar ester group.

Zimina et al. [15] characterized PS- $b$-PtBMA using LCCC. Under the critical conditions for the PtBMA block they were able to examine the length of the PS block in the block copolymers by using different binary solvent mixtures and different PS standards. Critical conditions for the PtBMA block were established in acetonitrile/dichloromethane and THF/dichloromethane.
Raust et al. [16] developed a 2D-LC method for complex (meth)acrylate-based copolymers synthesized by freeradical copolymerization, that is, isobutyl acrylate, isobutyl methacrylate, isobornyl acrylate, isobornyl methacrylate, and 2-etylhexyl acrylate. In the first dimension the CCD of the copolymers was determined using gradient chromatography on a reversed-phase column in the acetonitrile/THF mobile phase and in the second dimension the molarmass characteristics of the fractions eluted from the first dimension were determined by fast SEC in THF.

Falkenhagen et al. [17] determined the lengths of both blocks in PMMA- $b$-PtBMA copolymers using reversedphase and normal-phase LCCC.

The aim of our work was to chromatographically evaluate a mixture of three linear PS- $b$-P $t$ BMA copolymers consisting of polystyrene (PS) and poly ( $t$-butyl methacrylate) (PtBMA) blocks. The PS- $b$-P $t$ BMA copolymers were synthesized using living anionic polymerization and are well defined with respect to their molecular and structural characteristics. For a powerful 2D-LC characterization of the PS- $b$-P $t$ BMA mixture a sufficiently high resolution in the first dimension is essential. Therefore, much of our work was dedicated to a determination of the chemical composition distribution using different liquid-chromatographic techniques (gradient LC and LCCC) and different stationary phases (C18 and C8 reversed phase and $\mathrm{SiO}_{2}$ normal phase). The optimized chromatographic conditions in gradient liquid chromatography were established by studying the elution behaviour of the PS and $\mathrm{P} t \mathrm{BMA}$ homopolymers under different gradients. For the LCCC measurements a series of PS homopolymers of different molar masses and uniform molar-mass distributions were tested in different mobile phase compositions. Both LC methods were finally hyphenated with high-speed SEC in the second dimension.

\section{Experimental Methods}

\subsection{Materials}

2.1.1. Block Copolymers. The PS- $b$-PtBMA copolymers were synthesized in the laboratory of Professor Dr. Nikos Hadjichristidis, Industrial Chemistry Laboratory, Polymers Group, University of Athens [18]. The copolymers are of low-molar-mass dispersity and with a defined length of both blocks. The number average molar mass of the PS block is about $29500 \mathrm{~g} / \mathrm{mol}$ in all the samples, whereas that of the PtBMA block increases in the order copolymer 1 $(3000 \mathrm{~g} / \mathrm{mol})<$ copolymer $2(7000 \mathrm{~g} / \mathrm{mol})<$ copolymer 3 $(32000 \mathrm{~g} / \mathrm{mol})$.

2.1.2. Solvents. The solvents used for the gradient LC on the reversed-phase column were acetonitrile (ACN gradient grade; Sigma Aldrich, Germany) and tetrahydrofuran (THF p.a.; Merck, Germany). The solvents used for the gradient LC on the normal-phase column were $n$-hexane (p.a.; Merck, Germany) and THF. All the solvents were filtered through a Millipore filter system $(0.45 \mu \mathrm{m}$, Nylon 66, Supelco) and degassed with a Brason 2210 ultrasonic degasser. 
2.2. NMR. The ${ }^{1} \mathrm{H}$ NMR spectra of the PS- $b$-P $t$ BMA samples were recorded on a $300-\mathrm{MHz}$ Unity Inova 300 spectrometer (Varian) in the pulse Fourier Transform mode with a relaxation delay of $5 \mathrm{~s}$ and an acquisition time of $3 \mathrm{~s}$. Tetramethylsilane $\left(\mathrm{Me}_{4} \mathrm{Si}, \delta=0\right)$ was used as an internal chemical-shift standard. The PS- $b$-PtBMA copolymers were dissolved in $\mathrm{CDCl}_{3}-d_{1}$ as a solvent. Trifluoroacetic acid was added to solutions of block copolymers in order to shift the peak of the water in chloroform towards a lower magnetic field. The spectra were recorded immediately after the addition of the acid to avoid the hydrolysis of the $t$-butyl ester groups.

2.3. Size Exclusion Chromatography Coupled to a Multiangle Light Scattering Photometer (SEC-MALS). The SEC-MALS measurements were performed at room temperature using a Hewlett-Packard pump series 1100 coupled to a DAWN HELEOS laser photometer with a GaAs linearly polarized laser $\left(\lambda_{0}=658 \mathrm{~nm}\right)$ and to an Optilab rEX interferometric refractometer (RI) operating at the same wavelength as the photometer (both instruments are from Wyatt Technology Corp., USA). The separations were carried out using a $5 \mu \mathrm{m}$ Resipore SDV column with a precolumn $(300 \mathrm{~mm}$ length and $7.5 \mathrm{~mm}$ i.d., Polymer Laboratories) in THF. The Resipore SDV column exhibits a wide pore-size distribution, covering the molar masses from 200 to $400,000 \mathrm{Da}$. The nominal eluent flow rate was $1.0 \mathrm{~mL} / \mathrm{min}$. The mass of the samples injected onto the column was typically $3 \times 10^{-4} \mathrm{~g}$, whereas the solution concentration was $3 \times 10^{-3} \mathrm{~g} / \mathrm{mL}$. The calculation of the molar-mass averages from MALS requires a sample-specific refractive-index increment $(d n / d c)$, which was determined from the known weight fractions of the PS and PtBMA blocks, which were determined based on proton NMR results (Table 1) and known refractive-index increments of the corresponding homopolymers in THF $(0.184 \mathrm{~mL} / \mathrm{g}$ for PS and $0.065 \mathrm{~mL} / \mathrm{g}$ for PtBMA). For the data acquisition and evaluation the Astra 5.3.4 software (Wyatt Technology Corp., USA) was utilized.

\subsection{HPLC}

2.4.1. Gradient $L C \times S E C$. The retention behaviour on reversed-phase (C18 and $\mathrm{C} 8$ ) columns was studied using a series of narrow-molar-mass dispersity PtBMA standards $(10,000$ and $30,000 \mathrm{~g} / \mathrm{mol})$ and PS standards $(10,300$ and $29,510 \mathrm{~g} / \mathrm{mol}$ ) at a solution concentration of $2 \mathrm{mg} / \mathrm{mL}$ in THF. On the normal-phase column the retention behaviour was studied using a series of PS standards $(29,510$ and $50,000 \mathrm{~g} / \mathrm{mol})$ and PtBMA standards $(10,000$ and $30,000 \mathrm{~g} / \mathrm{mol}$ ) at a solution concentration of $2 \mathrm{mg} / \mathrm{mL}$ in THF. A sample mixture was prepared by mixing stock solutions of each block copolymer with a concentration of $2 \mathrm{mg} / \mathrm{mL}$ in THF in a volume ratio of $1: 1: 1$.

For the reversed-phase gradient LC experiments LC Nucleosil C18 column $(250 \mathrm{~mm} \times 4 \mathrm{~mm}$ I.D., MachereyNagel $\mathrm{GmbH}$ ) and Zorbax Eclipse XDB-C8 (150 mm $\times 4.6 \mathrm{~mm}$ I.D., Agilent Technologies) were used. For the normal-phase gradient LC experiments a Nucleosil-Si
$(250 \mathrm{~mm} \times 4 \mathrm{~mm}$ I.D., Macherey-Nagel $\mathrm{GmbH})$ was used. The flow rate in the first dimension was $0.5 \mathrm{~mL} / \mathrm{min}$, while in the 2D-LC experiments it was reduced to $0.04 \mathrm{~mL} / \mathrm{min}$. In the second dimension a PSS SDV high-speed column $(50 \mathrm{~mm}$ $\times 20 \mathrm{~mm}$ I.D., Polymer Standards Service $\mathrm{GmbH}$ ) was used, and the THF flow rate was set to $3.0 \mathrm{~mL} / \mathrm{min}$. The PSS highspeed SDV column exhibits a wide pore-size distribution, covering the molar masses from 500 to $60.000 \mathrm{Da}$. The SEC column was calibrated using polystyrene standards of a narrow-molar-mass distribution with a concentration of $1 \mathrm{mg} / \mathrm{mL}$. For the 2D-LC experiments a Perkin Elmer series 200 and Agilent 1100 pumps and an evaporative lightscattering detector ELS 1000 (Polymer Laboratories) were used. For the data acquisition and evaluation a WinGPC v. 7 (Polymer Standards Service $\mathrm{GmbH}$ ) was utilized. The fractions were transferred from the first to the second dimension using an eight-port transfer valve equipped with two $100 \mu \mathrm{L}$ sample loops (EHC8W type, VICI Valco Instruments) that was controlled by a $2 \mathrm{D}$ WinGPC v. 7 software module.

2.4.2. $L C C C \times S E C$. The critical conditions for PS on the normal-phase column were determined in the mobile phase $n$-hexane and THF at different volume ratios by injecting the PS standards of 2,100 g/mol, 51,000 g/mol, and $97,200 \mathrm{~g} / \mathrm{mol}$ at a solution concentration of $2 \mathrm{mg} / \mathrm{mL}$ THF. In these experiments we used a UV DAD Perkin Elmer series 200 as a detector at $\lambda=254 \mathrm{~nm}$. In the $2 \mathrm{D}$ LCCC $\times$ SEC experiments the flow rate in the first dimension was reduced to $0.1 \mathrm{~mL} / \mathrm{min}$, while the THF flow rate in the second dimension was $4.0 \mathrm{~mL} / \mathrm{min}$.

\section{Results and Discussion}

3.1. Composition of PS-b-PtBMA Copolymers. The molar ratio between the PS and PtBMA repeat units in the PS- $b$-P $t$ BMA copolymers was determined using ${ }^{1} \mathrm{H}$ NMR spectroscopy. The signals in the range from $7.24 \mathrm{ppm}$ to $6.28 \mathrm{ppm}$ correspond to the PS phenyl ring (A). The signals in the range from $2.38 \mathrm{ppm}$ to $0.63 \mathrm{ppm}$ belong to the PS- $b$ PtBMA backbone, that is, three protons to the PS backbone (methylene group $\mathrm{C}$ and methine group $\mathrm{B}$ ) and two protons to the PtBMA backbone (methylene group $\mathrm{D}$ ), and to the methyl (E) and $t$-butyl side groups (F) of the PtBMA block. The ${ }^{1} \mathrm{H}$ NMR spectra of the PS- $b$-P $t$ BMA samples denoted by 1 and 3 show the presence of minute amounts of residual $t$ BMA monomer with the following signals: ${ }^{1} \mathrm{H}$ $\operatorname{NMR}\left(\mathrm{CDCl}_{3}\right) \delta(\mathrm{ppm}) 6.06\left(1 \mathrm{H}, \mathrm{dq}, J_{1,3}=1 \mathrm{~Hz}, J_{1,2}=\right.$ $1.5 \mathrm{~Hz}), 5.58\left(1 \mathrm{H}, \mathrm{dq}, J_{1,2}=1.5 \mathrm{~Hz}, J_{2,3}=1.5 \mathrm{~Hz}\right), 1.90(3 \mathrm{H}$, $\left.\mathrm{dd}, J_{1,3}=1 \mathrm{~Hz}, J_{2,3}=1.5 \mathrm{~Hz}\right), 1.52(9 \mathrm{H}, \mathrm{s})$ (Figure 1).

The molar ratio between the PS and PtBMA repeat units was determined by comparing the integral of the PS aromatic protons in the between region 7.24-6.28 with the integral of the PtBMA protons, taking into account that at the equimolar ratio the ratio between them is $5: 14$. The integral for the PtBMA protons was obtained from the integral ranging from 0.63 to $2.38 \mathrm{ppm}$ by subtraction of the integral for the protons of the PS backbone (C and D) and the integral 
TABLE 1: Molar-mass characteristics of the PS- $b$-PtBMA copolymers as determined by SEC-MALS.

\begin{tabular}{lcccccccc}
\hline $\begin{array}{l}\text { Sample } \\
\text { PS- } b \text {-P } t \text { BMA }\end{array}$ & $M_{w} \times 10^{-3} \mathrm{~g} / \mathrm{mol}$ & $M_{n} \times 10^{-3} \mathrm{~g} / \mathrm{mol}$ & $M_{w} / M_{n}$ & ${ }^{1} \mathrm{~mol}_{\mathrm{PS}}$ & ${ }^{2} w_{\mathrm{PS}}$ & ${ }^{3} d n / d c(\mathrm{~mL} / \mathrm{g})$ & $\begin{array}{c}M_{n} \times 10^{-3} \mathrm{PS} \\
\text { block g/mol }\end{array}$ & $\begin{array}{c}M_{n} \times 10^{-3} \mathrm{P} t \mathrm{BMA} \\
\mathrm{block} g / \mathrm{mol}\end{array}$ \\
\hline 1 & 32.0 & 31.0 & 1.03 & 0.937 & 0.916 & 0.174 & 29.0 & 2.0 \\
2 & 36.6 & 35.6 & 1.03 & 0.848 & 0.804 & 0.161 & 30.2 & 5.4 \\
3 & 62.0 & 60.4 & 1.03 & 0.562 & 0.484 & 0.123 & 33.9 & 26.5 \\
\hline
\end{tabular}

${ }^{1}$ molps: the mol fraction of PS was determined from proton NMR spectra.

${ }^{2} w_{\text {PS }}$ : the weight fraction of PS was determined from the known mol fraction of PS and the molar mass of PS unit.

${ }^{3} d n / d c$ was determined according to (1).

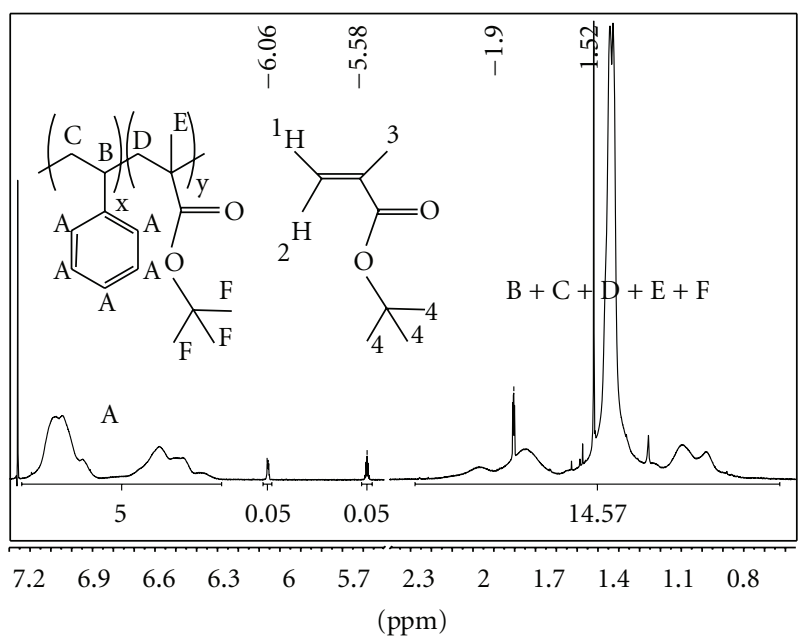

Figure 1: ${ }^{1} \mathrm{H}$ NMR spectrum of the PS- $b$-PtBMA copolymer number 3.

of the protons of the methyl (4) and $t$-butyl (F) groups of the $t$ BMA monomer. In the calculations we neglected the copolymers' terminal groups since the PS- $b$-PtBMA samples have high molar masses. The molar ratios between the PS and $\mathrm{P} t \mathrm{BMA}$ repeat units in the copolymers were $1: 0.067$ for sample $1,1: 0.179$ for sample 2 , and $1: 0.779$ for sample 3 .

From the molar ratios between the PS and PtBMA repeat units in the copolymers we calculated the corresponding weight fractions ( $\left.w_{\mathrm{PS}}, w_{\mathrm{P} t \mathrm{BMA}}\right)$, which were then used for the calculation of the copolymers' refractive-index increments $(d n / d c$, equation (1)). Consequently, these values were applied for the determination of the absolute molar-mass averages and the molar-mass distributions of the copolymers from the MALS photometer (Table 1):

$$
\frac{d n}{d c}=\left(\frac{d n}{d c}\right)_{\mathrm{PS}} w_{\mathrm{PS}}+\left(\frac{d n}{d c}\right)_{\mathrm{P} t \mathrm{BMA}} w_{\mathrm{P} t \mathrm{BMA}},
$$

where $(d n / d c)_{\mathrm{PS}}$ and $(d n / d c)_{\mathrm{P} t \mathrm{BMA}}$ are the refractive-index increments of the corresponding homopolymers in THF $\left(0.184 \mathrm{~mL} / \mathrm{g}\right.$ for PS and $0.065 \mathrm{~mL} / \mathrm{g}$ for PtBMA), while $w_{\mathrm{PS}}$ and $w_{\mathrm{P} t \mathrm{BMA}}$ are the weight fractions of the PS and PtBMA repeat units in the copolymers, respectively.

3.2. Molar-Mass Averages and Molar-Mass Distribution of PS$b$-PtBMA Copolymers. The SEC-MALS curves of the PS$b$-PtBMA copolymers are symmetrical (Figure 2 ), and the calculated molar-mass dispersities $\left(D_{M} \sim 1.03\right)$ indicate the very narrow-molar-mass distribution of the copolymers (Table 1). The molar-mass averages decrease in the order: copolymer $3>$ copolymer $2>$ copolymer 1 . All three copolymers have comparable lengths of the PS block, while the length of the PtBMA block decreases in the same order as the copolymers' molar-mass averages (Table 1). The copolymers 1 and 2 have very short PtBMA blocks, when compared to that of the PS block. In addition, the difference in the PtBMA block length in these two copolymers is small (Table 1 and Figure 3).

3.3. Reversed-Phase Liquid Chromatography of PS- $b-P t B M A$ Copolymers. The mixture of three copolymers was analysed using gradient HPLC on C18 and C8 reversed stationary phase columns, which is a frequently used chromatographic method for the separation of copolymers based on their chemical composition [19]. By changing the composition of the mobile phase during the run the solvent power of the mobile phase is adjusted to control the solubility and/or adsorption of the individual sample's constituents so that the CCD of the copolymers can be determined. In order to optimize the chromatographic conditions for the efficient separation of the PS- $b$-PtBMA copolymers, we first studied the elution behaviour of the individual homopolymers (PS and PtBMA) with a narrow-molar-mass distribution in the binary mobile phase, consisting of highand medium-polarity solvents, that is, acetonitrile (ACN) and THF. Starting with the higher content of the ACN as a nonsolvent for homopolymers and gradually raising the amount of THF in the mobile phase as a thermodynamically good solvent for homopolymers, elution according to Martin's rule was achieved (Figures 4(a) and 4(b)). The homopolymer with a higher molar mass was eluted after the homopolymer with the lower mass. The elution volume of the PtBMA homopolymer was slightly lower than that of the PS homopolymer of comparable molar mass, due to the higher polarity of the PtBMA, compared to the PS homopolymer. Martin's rule correlates the retention factor with the number of repeat units in the homopolymer in a semiempirical relation. The retention factor $\left(k^{\prime}\right)$ increases exponentially with the number of homopolymer repeat units (n) according to the following

$$
\ln k^{\prime}=\ln A+B n,
$$




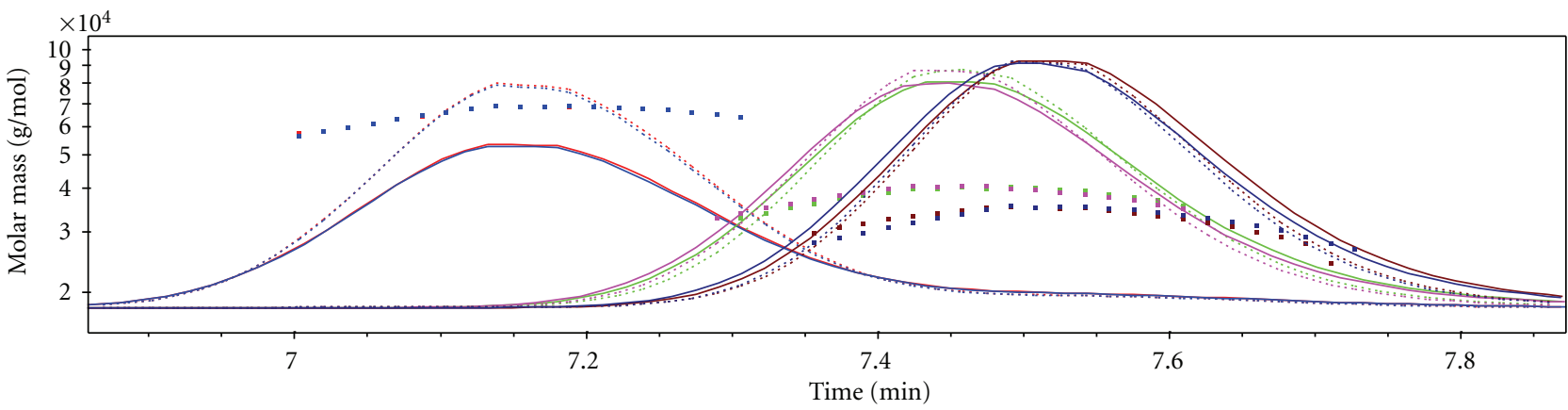

FIGURE 2: SEC-MALS chromatograms (the dashed lines indicate the DRI response, the dotted lines indicate the LS response at $90^{\circ}$ angle) and molar mass versus elution volume curves for the PS- $b$-PtBMA copolymers. From left to right: copolymer 3, copolymer 2, and copolymer 1.

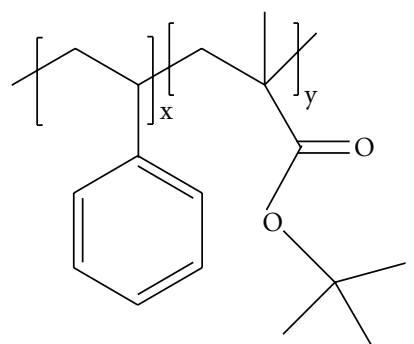

FIGURE 3: Schematic structure of PS- $b$-PtBMA; copolymer 1: $x=$ $279, y=14$; copolymer $2: x=290, y=38$; copolymer $3: x=$ $326, y=187$.

where $A$ and $B$ are empirical constants. The relation between $\ln k^{\prime}$ and the chain length, that is, the number of repeat units, $n$, is linear [20].

The overlay of chromatograms obtained with the gradient elution (ACN/THF) of the individual block copolymers and their mixture on the reversed-phase C18 column show that the peak eluted at a small elution volume in the chromatogram of the mixture belongs to the coeluted block copolymers 1 and 2, while the peak at a larger elution volume represents the block copolymer 3, which has the longest PtBMA block (Figure 5(a)).

Although we varied the chromatographic conditions by changing the gradient and the temperature of the measurements (Figure 5(b)) we were not able to separate the block copolymers 1 and 2 to the baseline, most probably due to the relatively short PtBMA block, when compared to the length of the PS block in the copolymers. At a higher temperature, that is, $50^{\circ} \mathrm{C}$, the block copolymers eluted at slightly lower elution volumes, but without any improvement in resolution (Figure 5(b)). Glöckner and Wolf showed that the elution of the PS- $b$-PtBMA copolymers on the reversed-phase C18 column was governed mainly by the solubility/precipitation mechanism, while on the phenyl column it was the adsorption mechanism [14].

The elution of the copolymers PS- $b$-P $t$ BMA was also performed on the C8 stationary phase, since we expected a better resolution, as block copolymers differ only in the length of the PtBMA block, which is slightly more polar than the PS block (Figures 6(a) and 6(b)).

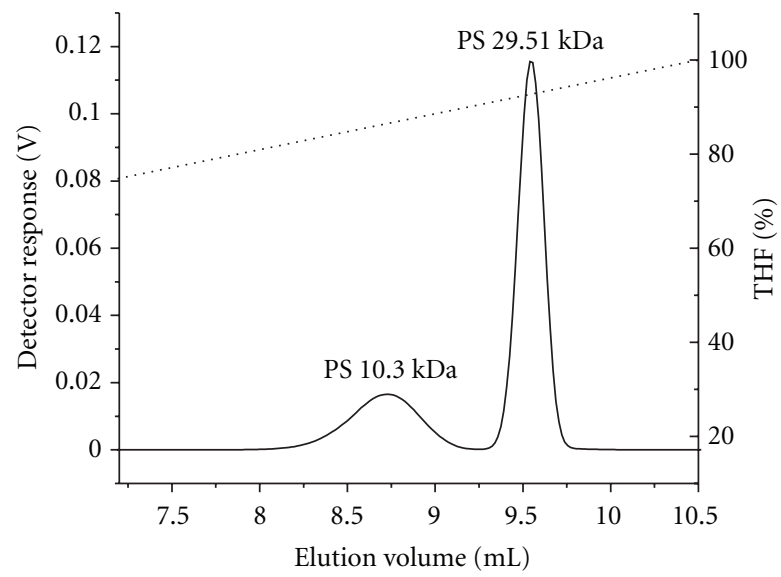

(a)

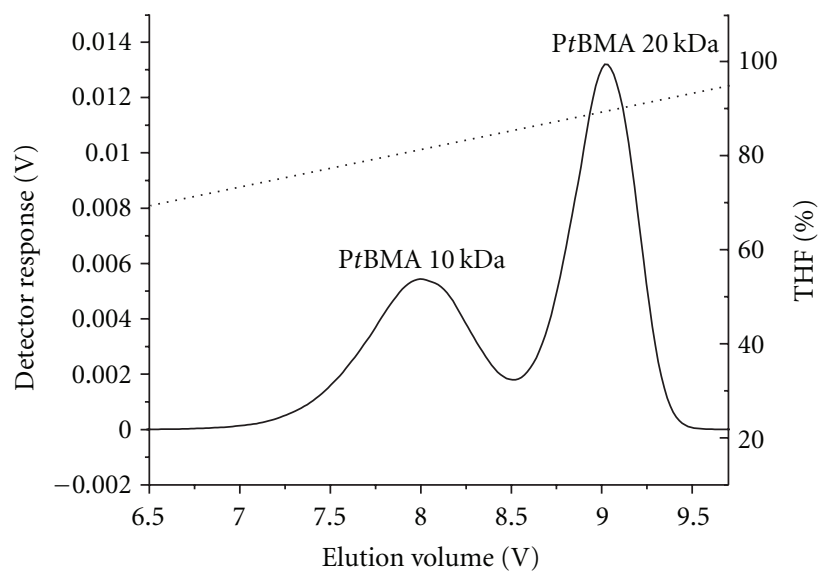

(b)

FIgure 4: Gradient separation of PS (a) and PtBMA (b) homopolymers on the reversed-phase C18 column in the ACN/THF mobile phase at a flow rate of $1 \mathrm{~mL} / \mathrm{min}$.

The PS- $b$-PtBMA copolymers elute from the C 8 column somewhat earlier than from the C18 column since the former stationary phase has a slightly higher polarity than the latter. The resolution between the PS- $b$-PtBMA samples 1 and 2 slightly improved, while that between the samples 2 and 


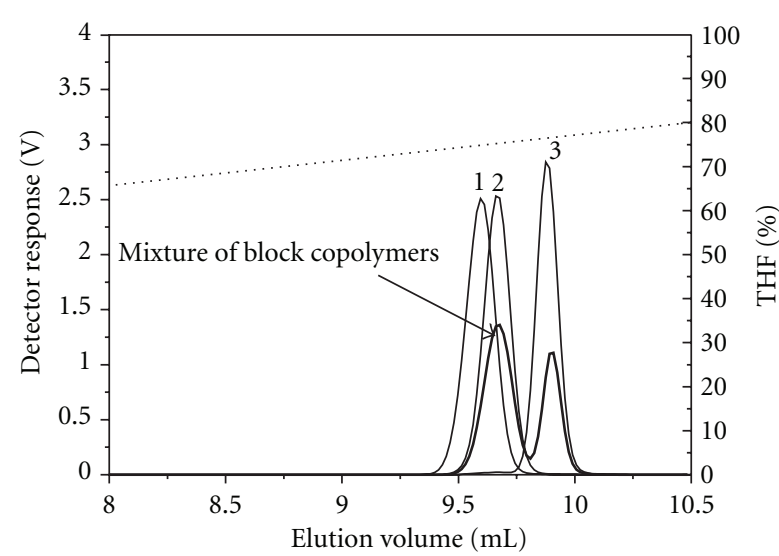

(a)

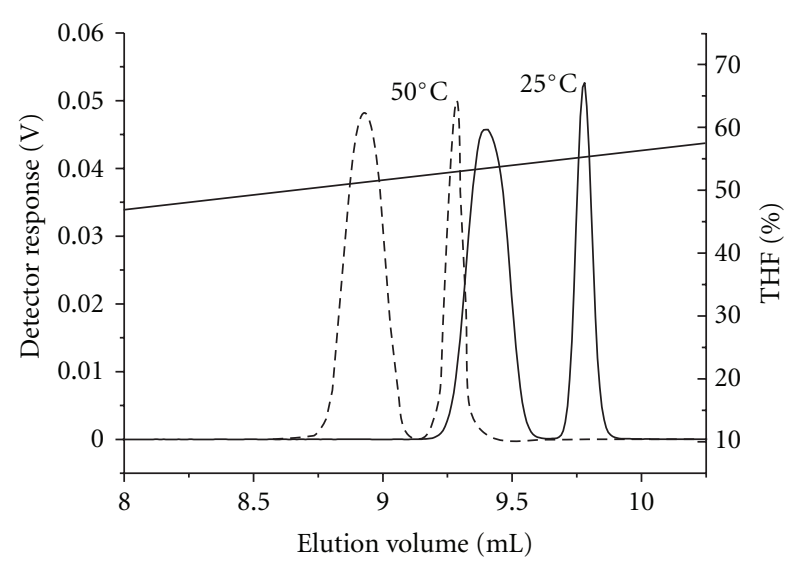

(b)

Figure 5: Overlay of the ELS curves of individual PS- $b$-P $t$ BMA copolymers and their mixture (a) and overlay of ELS curves of the mixture of block copolymers obtained at $50^{\circ} \mathrm{C}$ and $25^{\circ} \mathrm{C} \mathrm{(b) \text {; }}$ gradient ACN/THF, flow rate: $0.5 \mathrm{~mL} / \mathrm{min}$, column: Nucleosil C18.

3 worsened, compared to the results obtained on the C18 column (Figures 6(a) and 6(b)).

3.4. Normal-Phase Liquid Chromatography of PS-b-PtBMA Copolymers. For the normal-phase HPLC of the individual homopolymers and the PS- $b$-PtBMA copolymers we used the $n$-hexane/THF binary solvent gradient similar to the one used by Karanam et al. [21], who investigated the P $t$ BMA- $b$-PMMA- $b$-P $t$ BMA triblock copolymers. Since the polarity of the PtBMA is slightly higher than that of the PS we expected that the PtBMA homopolymers will elute on the normal-phase column at larger elution volumes than the PS homopolymers of comparable molar masses. Our results show the opposite elution order, that is, the PtBMA homopolymers eluted before the PS homopolymers (Figure 7(a)), which could be a consequence of the inaccessibility of the PtBMA ester carbonyl group for the interaction with the stationary phase due to the shielding effect of the bulky $t$ butyl side group. Overall, the results show that the separation of the copolymer mixture on the normal-phase column using the $n$-hexane/THF gradient (Figure $7(\mathrm{~b})$ ) is worse than that on the reversed-phase columns (Figure 6).

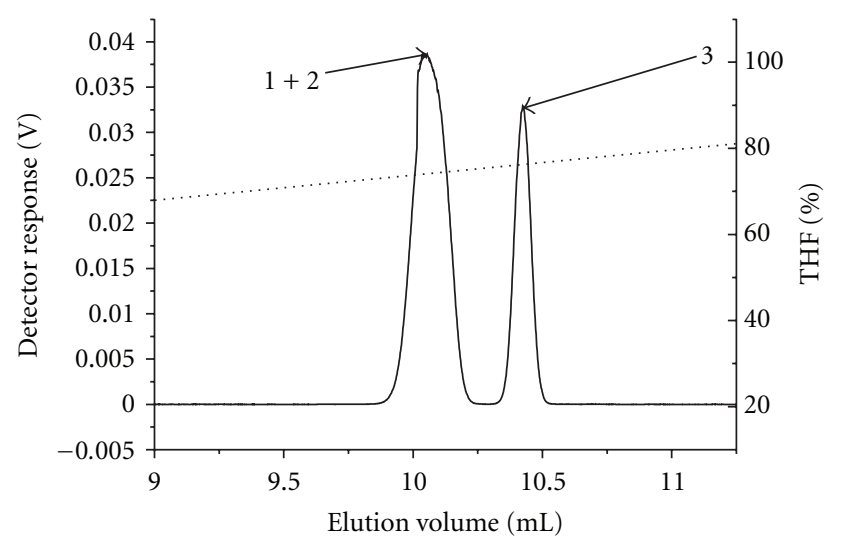

(a)

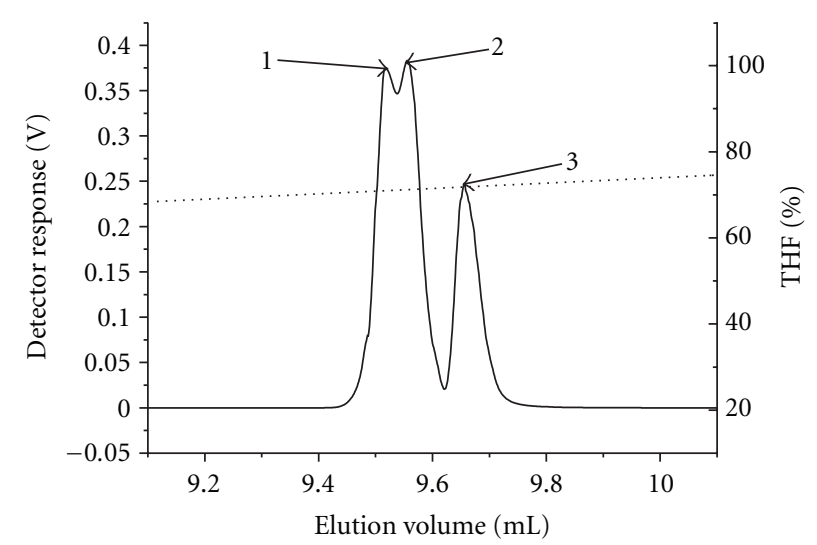

(b)

FIGURE 6: Gradient separation of the mixture of block copolymers on reversed-phase columns Nucleosil C18 (a) and Zorbax C8 (b) in $\mathrm{ACN} / \mathrm{THF}$ at a flow rate of $0.04 \mathrm{~mL} / \mathrm{min}$.

3.5. Liquid Chromatography of PS-b-PtBMA Copolymers under Critical Conditions. For block copolymers that are eluted under critical conditions for one block the other block defines the mode of elution, that is, the exclusion or adsorption mode. Under critical conditions for the PS block the elution of the PS- $b$-PtBMA copolymers is governed only by the molar mass of the PtBMA block. The critical conditions for PS on the normal-phase column were determined at $55^{\circ} \mathrm{C}$ by injecting PS standards of different molar masses using the $n$-hexane/THF mobile phase of different compositions. At the volume ratio of $n$-hexane/THF $=60 / 40$ the PS homopolymers eluted at the same elution volume regardless of their molar mass (Figure 8), which is in accordance with the literature data [8].

The separation of the mixture of PS- $b$-PtBMA copolymers under the critical conditions for the PS shows that the PtBMA block eluted under the exclusion mode (Figure 9). Unfortunately, the separation was less efficient than that using gradient LC on reversed-phase columns, which is most probably a consequence of the fact that the critical conditions for PtBMA ( $n$-hexane/THF $=57: 43$ ) [17] are close to those for PS, which indicate not much difference in the polarity of both constituting blocks. 


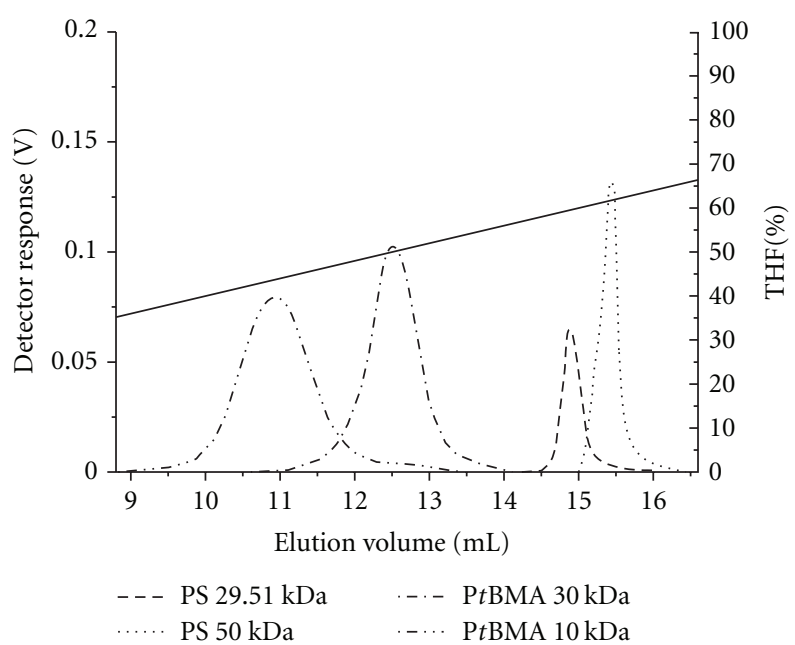

(a)

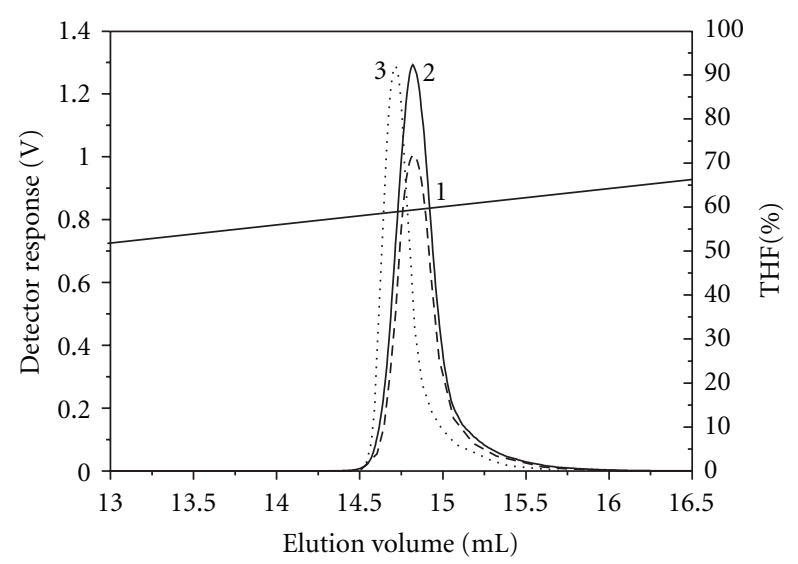

(b)

FIgURE 7: Overlay of ELS chromatograms of PS and PtBMA homopolymers (a) and individual block copolymers PS- $b$-P $t$ BMA (b); gradient $n$-hexane/THF, flow rate $0.5 \mathrm{~mL} / \mathrm{min}$, column: Nucleosil Si.

3.6. Two-Dimensional LC of PS- $b$-PtBMA Copolymers. Twodimensional experiments on PS- $b$-PtBMA copolymers were performed only under HPLC conditions giving the best separation of copolymers according to composition, that is, in the first dimension we used liquid chromatography on the reversed-phase C18 and C8 columns. In the second dimension we used the high-speed SEC column, which separates the copolymers according to molar mass. A separate SEC separation of the mixture of block copolymers on this column results in one broad peak with a shoulder.

In $2 \mathrm{D}$-LC, the $2 \mathrm{D}$ plot is presented as a contour plot in which the first dimension separation is represented along the $y$-axis (gradient LC, C18 or C8) and the second dimension (SEC) along the $x$-axis. The contour plot of the 2D-LC separation of the mixture of copolymers using the reversedphase C18 column in the first dimension (Figure 10) shows two baseline separated spots, among which the spot denoted with 2 represents the coeluted block copolymers 1 and 2 , whereas the spot denoted with 1 represents the block

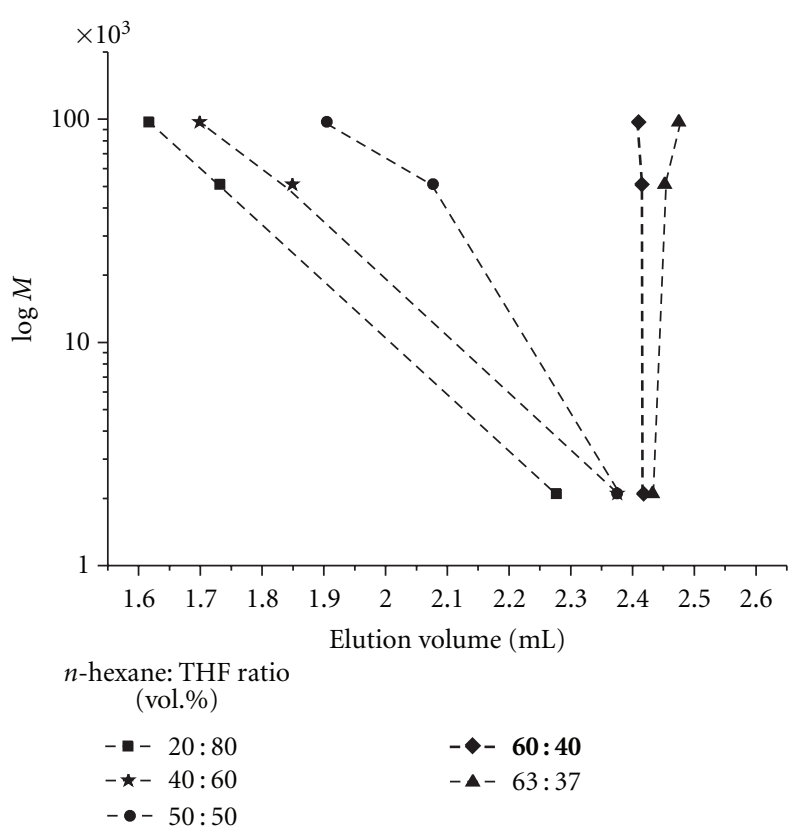

Figure 8: Determination of critical conditions for polystyrene on normal phase Nucleosil Si $300 \AA ̊$ column using a different composition of $n$-hexane/THF mobile phase at a temperature of $55^{\circ} \mathrm{C}$ and a flow rate of $0.5 \mathrm{~mL} / \mathrm{min}$. UV detector UV $(\lambda=254 \mathrm{~nm})$.

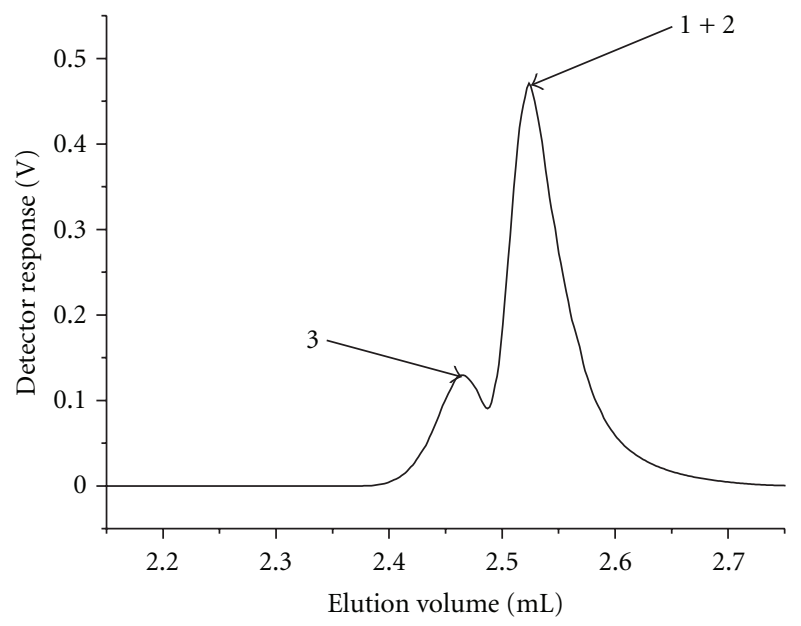

FIgURE 9: The chromatogram of the mixture of block copolymers obtained under critical conditions for polystyrene ( $n$-hexane/THF: $60 / 40, T=55^{\circ} \mathrm{C}$ ) on normal-phase Nucleosil Si $300 \AA$ Á column at a flow rate of $0.5 \mathrm{~mL} / \mathrm{min}$. ELSD detector.

copolymer 3 . Since in spot 2 two copolymers coeluted, it is broader along the $y$-axis in comparison with spot 1 .

A similar 2D-LC elution pattern of the block copolymers was obtained when we used the C8 instead of the C18 column in the first dimension (Figure 11), although a one-dimensional gradient HPLC separation on the C8 column gave partially separated peaks for the block copolymers 1 and 2 (Figure 6(b)). In addition, the intensity of the spots in the 2D-LC contour plot is lower when using the C8 instead of the $\mathrm{C} 18$ column. This suggests that part of the sample is retained 


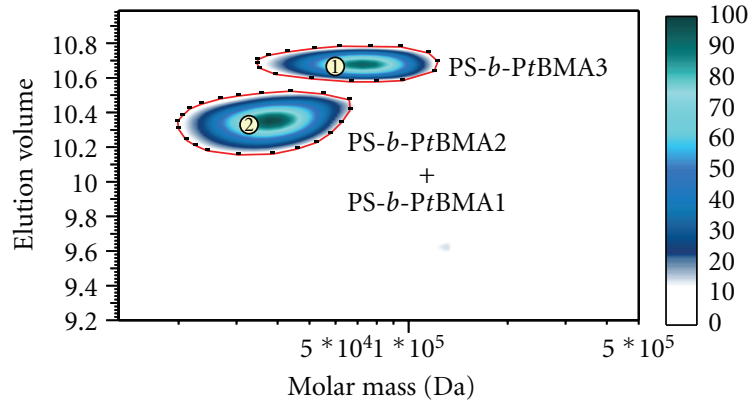

FIgURE 10: 2D-LC separation of the mixture of block copolymers. 1D: Nucleosil C18 column, gradient ACN/THF, $0.04 \mathrm{~mL} / \mathrm{min}$. 2D: PSS SDV High-Speed column, THF, $3 \mathrm{~mL} / \mathrm{min}$. ELSD detector.

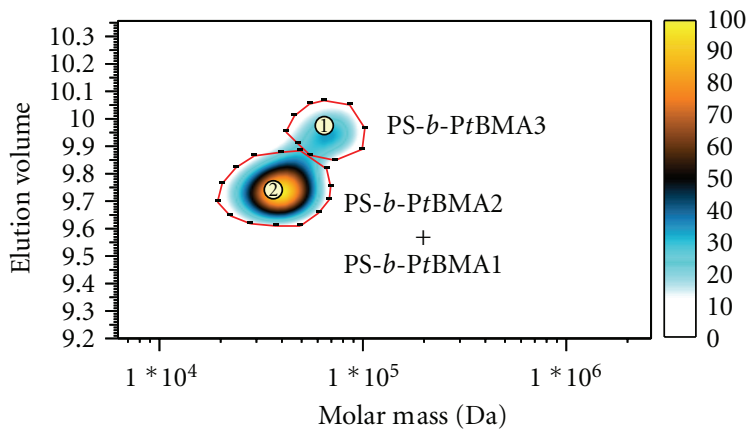

Figure 11: 2D-LC separation of the mixture of block copolymers. 1D: Zorbax C8, gradient ACN/THF, $0.04 \mathrm{~mL} / \mathrm{min}$. 2D: PSS SDV High-Speed, THF, $3 \mathrm{~mL} / \mathrm{min}$. ELSD detector.

TABLE 2: Molar-mass averages of the mixture of block copolymers determined by the 2D-LC and the calibration of SEC columns using PS standards.

\begin{tabular}{lcccc}
\hline \multirow{2}{*}{ Column } & \multicolumn{2}{c}{ Spot 1 (copolymer 3) } & \multicolumn{2}{c}{ Spot 2 (copolymers 1 and 2) } \\
& $M_{n}(\mathrm{kDa})$ & $M_{n}(\mathrm{kDa})$ & $M_{w}(\mathrm{kDa})$ & $M_{n}(\mathrm{kDa})$ \\
\hline Nucleosil C18 & 71.2 & 66.4 & 38.1 & 35.7 \\
Zorbax C8 & 67.0 & 64.7 & 39.5 & 37.1 \\
\hline
\end{tabular}

on the column, most probably due to the stronger interaction of the PS- $b$-PtBMA copolymers with the C8 rather than the C18 stationary phase, especially if the copolymer contains a longer PtBMA block (sample 3). Such interactions and the partial penetration of the solute into the narrow pores of the stationary phase, which result in a fully retained sample and a decreased sample recovery, were already reported in the literature $[22,23]$. The results of the molar-mass determination in the 2D-LC experiments indicate that the relative molar-mass values of the PS- $b$-P $t$ BMA copolymers are in relatively good agreement with the absolute ones and that spot two represents the average molar mass of the copolymers 1 and 2 (Table 2).

\section{Conclusion}

We studied the elution behaviour of a mixture of three PS$b$-PtBMA copolymers using various liquid-chromatography techniques. The PS- $b$-P $t$ BMA copolymers were of a defined structure with similar PS block lengths and different PtBMA block lengths. The composition of the mixture was studied by reversed-phase (C18 and C8) and normal-phase liquid chromatography and liquid chromatography under critical conditions for PS. The results showed that the most efficient separation of copolymers according to their composition was achieved using reversed-phase liquid chromatography. On the C 8 column the resolution between the PS- $b$-P $t$ BMA copolymers 1 and 2, which both consist of much shorter PtBMA block as compared to the length of PS block, slightly improved, while the resolution between the copolymer 2 and the copolymer 3 , which contains much longer PtBMA block, worsened, compared to the results obtained on the C18 column. Since most promising separation according to copolymer composition was obtained by reversed-phase LC technique, it was used as a method for the composition separation of copolymers in the first dimension of $2 \mathrm{D}$ LC experiments. The results of the $2 \mathrm{D}$ chromatographic separation revealed that none of the techniques used was able to completely separate the copolymer mixture, which was ascribed to the much shorter length of the PtBMA blocks, compared to the PS block in the two block copolymers of the mixture and the small difference in the PtBMA block length in these PS- $b$-P $t$ BMA copolymers.

\section{Acknowledgments}

The authors gratefully acknowledge the financial support of the Ministry of Higher Education, Science and Technology of the Republic Slovenia (program P2-0145) and Professor Dr. Nikos Hadjichristidis for providing them with block copolymer samples.

\section{References}

[1] N. Hadjichristidis, S. Pispas, and G. A. Flouda, Block Copolymers Synthetic Strategies, Physical Properties and Applications, John Wiley \& Sons, Hoboken, NJ, USA, 2003.

[2] H. Pasch and B. Thrathnigg, HPLC of Polymers, Springer, Berlin, Germany, 1999.

[3] D. Berek, "Two-dimensional liquid chromatography of synthetic polymers," Analytical and Bioanalytical Chemistry, vol. 396, no. 1, pp. 421-441, 2010.

[4] A. M. Striegel, W. Y. Wallace, J. J. Krikland, and D. D. Bly, Modern Size-Exclusion Liquid Chromatography, John Wiley \& Sons, Hoboken, NJ, USA, 2nd edition, 2009.

[5] S. J. Kok, T. H. Hankemeier, and P. J. Schoenmakers, "Comprehensive two-dimensional liquid chromatography with online fourier-transform-infrared-spectroscopy detection for the characterization of copolymers," Journal of Chromatography A, vol. 1098, no. 1-2, pp. 104-110, 2005.

[6] P. Kilz, "Two-dimensional chromatography as an essential means for understanding macromolecular structure," Chromatographia, vol. 56, pp. 3-14, 2004.

[7] P. Kilz, Copolymer Analysis by LC Methods, Including TwoDimensional Chromatography, Encyclopedia of Chromatography, Marcel Dekker, 2002.

[8] A. Horst and P. J. Schoenmakers, "Comprehensive twodimensional liquid chromatography of polymers," Journal of Chromatography A, vol. 1000, no. 1-2, pp. 693-709, 2003. 
[9] A. Greiderer, L. Steenken, T. Albers, G. Truyols, and P. Schoenmakers, "Characterization of hydroxypropylmethylcellulose (HPMC) using comprehensive two-dimensional liquid chromatography," Journal of Chromatography A, vol. 1218, no. 34, pp. 5787-5793, 2011.

[10] H. Pasch, K. Mequanint, and J. Adrian, "Two-dimensional chromatography of complex polymers. 3. Full analysis of polystyrene-poly(methyl methacrylate) diblock copolymers," e-Polymers, no. 005, 2002.

[11] A. Ginzburg, T. Macko, V. Dolle, and R. Brüll, "Characterization of polyolefins by comprehensive high-temperature twodimensional liquid chromatography (HT 2D-LC)," European Polymer Journal, vol. 47, no. 3, pp. 319-329, 2011.

[12] H. Mori and A. H. E. Müller, "New polymeric architectures with (meth)acrylic acid segments," Progress in Polymer Science, vol. 28, no. 10, pp. 1403-1439, 2003.

[13] G. Glöckner and A. H. E. Müller, "Gradient high-performance liquid chromatography of statistical and block copolymers of styrene and t-butyl methacrylate," Journal of Applied Polymer Science, vol. 38, no. 9, pp. 1761-1774, 1989.

[14] G. Glöckner and D. Wolf, "Gradient high performance liquid chromatography of block copolymers of styrene and t-butyl methacrylate. II. Association phenomena in mixtures of block copolymer with polystyrene," Chromatographia, vol. 34, no. 58, pp. 363-368, 1992.

[15] T. M. Zimina, J. J. Kever, E. Y. Melenevskaya, and A. F. Fell, "Analysis of block copolymers by high-performance liquid chromatography under critical conditions," Journal of Chromatography, vol. 593, no. 1-2, pp. 233-241, 1992.

[16] J.-A. Raust, A. Brüll, C. Moire, C. Farcet, and H. Pasch, "Two-dimensional chromatography of complex polymers. 6. Method development for (meth)acrylate-based copolymers," Journal of Chromatography A, vol. 1203, no. 2, pp. 207-216, 2008.

[17] J. Falkenhagen, H. Much, W. Stauf, and A. H. E. Müller, "Characterization of block copolymers by liquid adsorption chromatography at critical conditions. 1. Diblock copolymers," Macromolecules, vol. 33, no. 10, pp. 3687-3693, 2000.

[18] N. Hadjichristidis, H. Iatrou, S. Pispas, and M. Pitsikalis, "Anionic polymerization: high vacuum techniques," Journal of Polymer Science A, vol. 38, no. 18, pp. 3211-3234, 2000.

[19] G. Glöckner, Gradient HPLC of Copolymers and Chromatographic Cross-Fractionation, Springer, New York, NY, USA, 1991.

[20] A. Skvortsov and B. Trathnigg, "Martin's rule revisited. Its molecular sense and limitations," Journal of Chromatography A, vol. 1015, no. 1-2, pp. 31-42, 2003.

[21] S. Karanam, H. Goossens, B. Klumperman, and P. Lemstra, "'Controlled' synthesis and characterization of model methyl methacrylate/tert-butyl methacrylate triblock copolymers via ATRP," Macromolecules, vol. 36, no. 9, pp. 3051-3060, 2003.

[22] D. Berek and A. Russ, "Limited sample recovery in coupled methods of high-performance liquid chromatography of synthetic polymers," Chemical Papers, vol. 60, no. 3, pp. 249252, 2006.

[23] D. Berek, "Separation of parent homopolymers from diblock copolymers by liquid chromatography under limiting conditions of desorption 3. Role of column packing," Polymer, vol. 51, no. 3, pp. 587-596, 2010. 


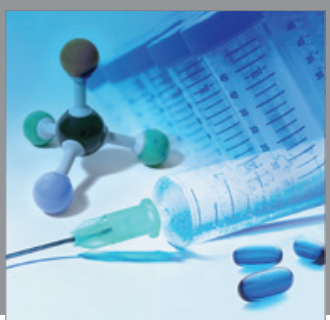

International Journal of

Medicinal Chemistry

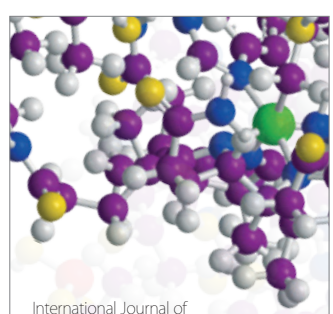

Carbohydrate Chemistry

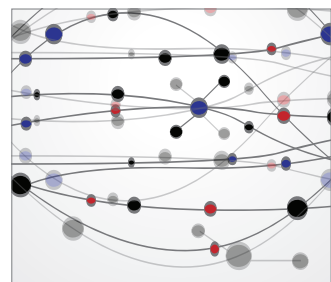

The Scientific World Journal
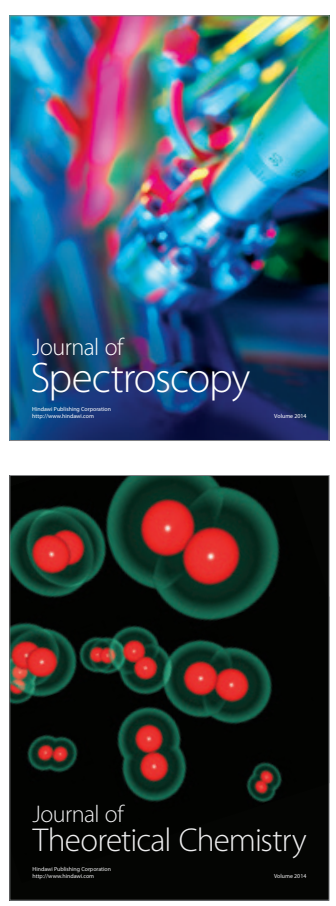
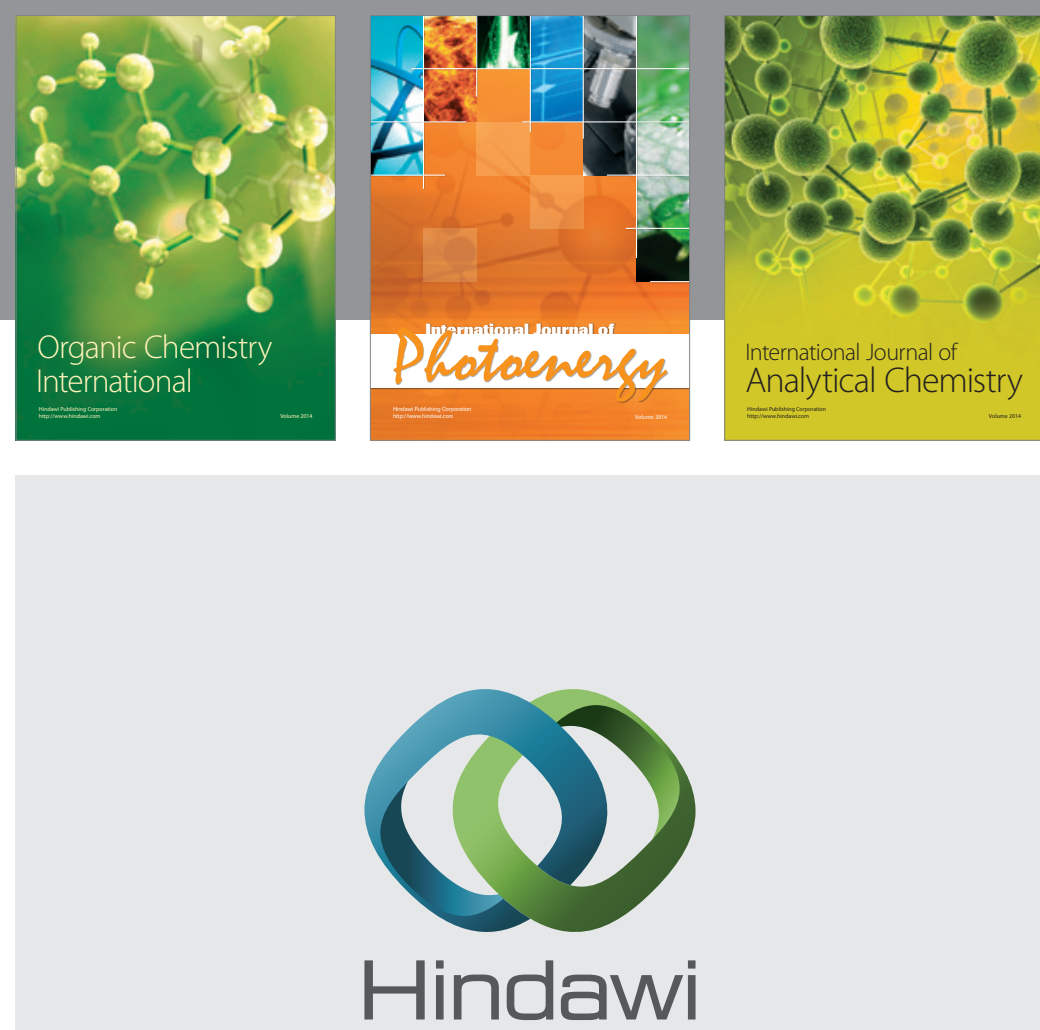

Submit your manuscripts at

http://www.hindawi.com
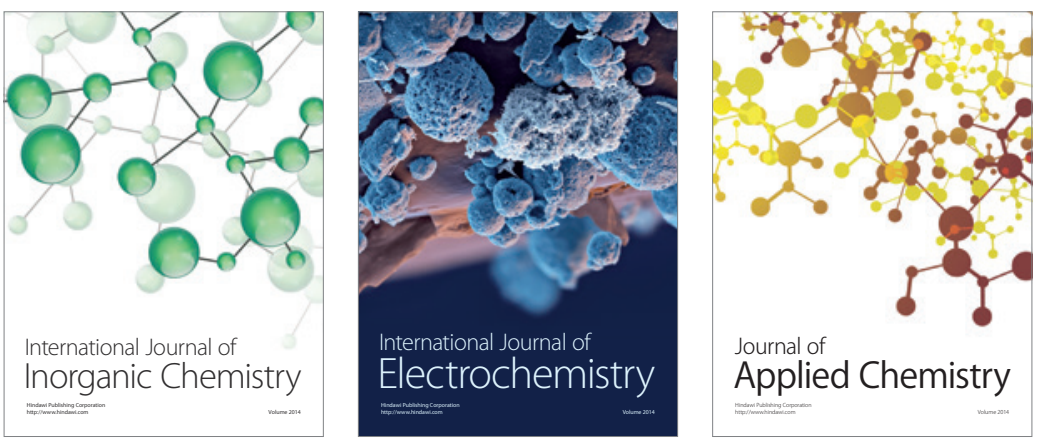

Journal of

Applied Chemistry
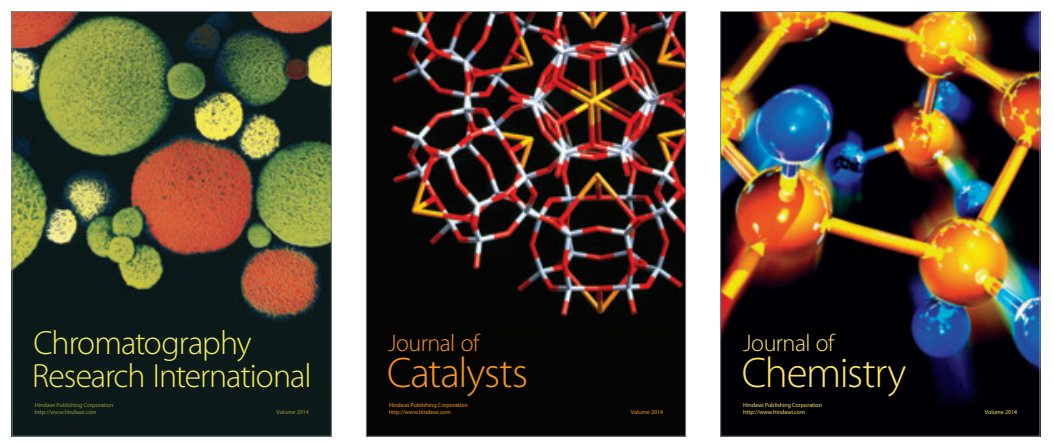
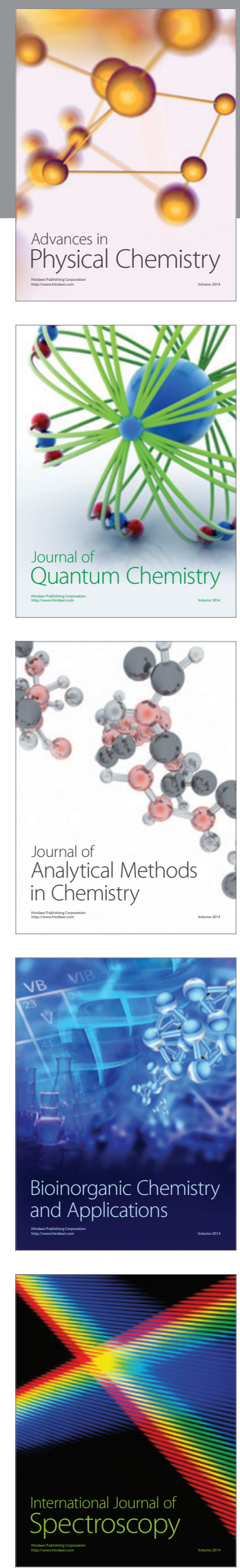